\title{
Sensitivity Analysis of the Tetrapolar Electrical Impedance Measurement Systems Using COMSOL Multiphysics for the non-uniform and Inhomogeneous Medium
}

\author{
Onic Islam Shuvo and Md. Naimul Islam* \\ *Department of Physics, Dhaka University, Dhaka-1000, Bangladesh.
}

(Received: 20 April 2015; Accepted: 15 November 2015)

\begin{abstract}
One of the major problems with Electrical Impedance Tomography (EIT) is the lack of spatial sensitivity within the measured volume. In this paper, sensitivity distribution of the tetrapolar impedance measurement system was visualized considering a cylindrical phantom consisting of homogeneous and inhomogeneous medium. Previously, sensitivity distribution was analysed analytically only for the homogeneous medium considering simple geometries and the distribution was found to be complex ${ }^{1,2}$. However, for the inhomogeneous volume conductors sensitivity analysis needs to be done using finite element method (FEM). In this paper, the results of sensitivity analysis based on finite element method using COMSOL Multiphysics simulation software are presented. A cylindrical non-uniform, inhomogeneous phantom, which mimics the human upper arm, was chosen to do the experiments by varying different parameters of interest. A successful method for controlling the region of interest was found where the sensitivity was maximum. Refining the finite element mesh size and introducing multifrequency input current (up to $1 \mathrm{MHz}$ ) this simulation method can be further improved.
\end{abstract}

Key words: Tetrapolar, Impedance, Tomography, Mesh, Sensitivity distribution.

\section{Introduction}

Electrical Impedance Tomography (EIT) is a technique to visualize the spatial distribution of electrical conductivity inside an object. Electrical impedance measurements on the human body have been found a variety of applications in clinical diagnosis and research including the measurement of physiological function ${ }^{3}$, tissue characterisation ${ }^{4}$ and imaging $^{5}$. In EIT, usually an alternating current of about $1 \mathrm{~mA}$ is injected in one pair of electrodes and voltages are measured from the other pairs. Current injection is then moved between another, commonly adjacent pair of electrodes so that all electrode pairs are used (fig. 1.1). Several electrode configurations can be used in EIT; however, they are all based on tetrapolar measurements because of its ability to minimize the impact of electrodes' contact impedance on the measurements. The tetrapolar electrode configuration has been used in a number of research areas such as respiratory system ${ }^{6}$, cardiac system ${ }^{7}$, cervical neoplasia, tissue characterization ${ }^{4}$ etc. However, there is very little information available for the sources of errors, when making tetrapolar impedance measurements. The spatial sensitivity of tetrapolar impedance measurements is complex ${ }^{1}$ having regions of negative sensitivity, which may introduce large errors when measuring the impedance for any heterogeneous materials. Earlier, much of the works on sensitivity analysis were done analytically based on phantom experiments and simulation considering simple geometries $^{1,2}$.

The availability of COMSOL Multiphysics package has enabled us to get the numerical solution for a complex geometries using finite element method. Through finite element simulation we can obtain a large number of data within a certain range which is impossible to get through experimental techniques. Our aim was to conduct a computer simulation study by COMSOL Multiphysics in order to investigate the sensitivity distributions in a tetrapolar measurement system by applying the Gezelowitz lead field theory ${ }^{8}$. The purpose of the study was to gain further understanding of the problems in EIT measurement.
In this paper the simulation results conducted with a $3 \mathrm{D}$ model mimicking the anatomy of human upper hand is presented.

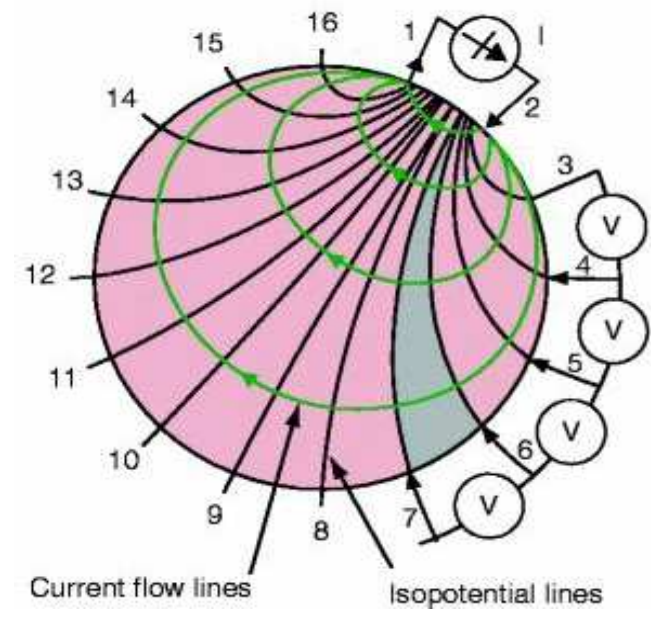

Fig. 1. 1. Diagram of a 16 electrode EIT system. Current, $I$, is imposed across the core $\Omega$ through a pair of adjacent electrodes while the voltage distribution, $V$ is measured between each set of neighbouring adjacent electrodes. After the voltage measurements around the entire perimeter, the current drive electrodes are rotated to the neighbouring electrode pair and the voltages at all electrodes are measured once again. This process continues until the 256 sets of voltage data are obtained.

\section{Sensitivity Analysis}

Sensitivity can be defined as the fractional change of transfer impedance (ratio of the measured potential and the applied current) with the change of conductivity inside a particular region. Considering the divergence theorem of Gauss in an arbitrary closed bounded region $V$, whose boundary, $\Omega$ is a piecewise smooth surface (fig. 2.1), the relationship between the measured boundary voltages and the conductivity distribution within the region $V$ can be calculated according to equation (2.1).

*Author for correspondence. e-mail: naimulphy@du.ac.bd 
$\int_{\Omega} \sigma(\nabla \varphi) \cdot d s=\int_{V} \sigma(\nabla \varphi \cdot \nabla \psi) d V$

where, $\varphi$ and $\psi$ are scalar potential functions, $d s$ is the unit vector directed outward normal to the boundary and the volume integral is taken over the entire bounded region.

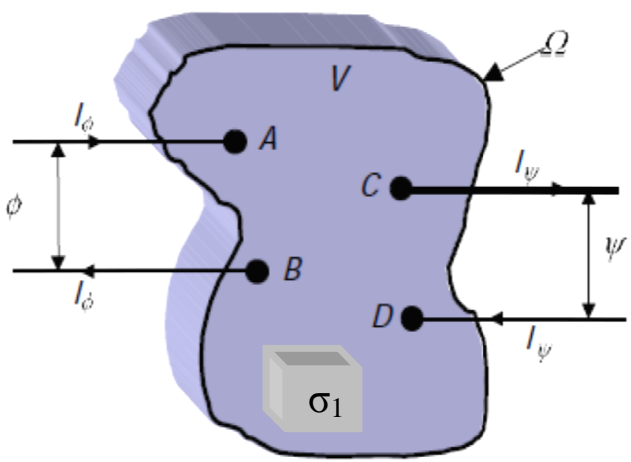

Fig. 2. 1. Volume conductor $V$ of conductivity $\sigma_{1}$ surrounded by air, where $A, B$ are current electrodes, $C, D$ are potential electrodes, and $\varphi$ are the potential fields caused by $\boldsymbol{I}_{\boldsymbol{\varphi}}$ and $\boldsymbol{I} \psi$, respectively.

If the region $V$ is surrounded by an insulating boundary $\Omega$ (fig. 2.1), the surface integral will be zero except at the electrodes' site where current is passing into and out of the region $V$ and will be given by

$\int_{\Omega} \sigma(\nabla \varphi) \cdot d \boldsymbol{s}=I_{\phi}\left(\psi_{A}-\psi_{B}\right)=I_{\phi} \psi_{A B}$

where, $I_{\varphi}$ is the drive current into the region $V$ through the electrodes $A$ and $B$. Similarly, when driving current $I_{\Psi}$ is inserted through electrodes $C$ and $D$, the surface integral will be equal to $I_{\psi} \varphi_{C D}$. Then, equation (2.1) can be rewritten as

$I_{\varphi} \psi_{A B}=\varphi_{C D}=\int_{V} \sigma(\nabla \varphi \cdot \nabla \psi) d V$

The equation (2.3) follows the reciprocity theorem, from which the transfer impedance can be defined and calculated using the equation (2.4).

$T_{Z}=\frac{\varphi_{C D}}{I_{\varphi}}=\frac{\Psi_{A B}}{I_{\Psi}}$

It can be noticed in equation (2.4) that reciprocity applies so that the drive and receive electrodes pair can be interchanged without changing the measured transfer impedance, $T_{Z}$. If the conductivity at an internal region of the volume conductor changes from $\sigma_{1}$ to $\sigma_{2}\left(=\sigma_{1}+\Delta \sigma\right)$, the potential field $\varphi$ will change from $\varphi$ to $\varphi^{\prime}(=\varphi+\Delta \varphi)$. Since the scalar potential remains unchanged, equation (2.1) can be combined to give-

$\int_{\Omega} \sigma\left(\varphi-\varphi^{\prime}\right)(\boldsymbol{\nabla} \psi) \cdot \boldsymbol{d} \boldsymbol{s}=\int_{V}\left(\sigma_{1}-\sigma_{2}\right)(\boldsymbol{\nabla} \phi \cdot \boldsymbol{\nabla} \psi) d V$

Considering volume conductor, the equation (2.5) becomes

$-\Delta \varphi_{C D}\left(-I_{\psi}\right)=-\int_{V} \Delta \sigma[\nabla(\varphi+\Delta \varphi) . \nabla \psi] d V$

Dividing each side by $I_{\varphi} I_{\psi}$, equation (2.6) can be rewritten to give the result obtained by Geselowitz ${ }^{8}$, such as
$\Delta Z=\frac{\Delta \varphi_{C D}}{I_{\varphi}}=-\int_{V} \Delta \sigma(x, y, z)\left[\nabla(\varphi+\Delta \varphi) \frac{1}{I_{\varphi}} \cdot \nabla \frac{1}{I_{\Psi}}\right] d V$

It should be noted that Geselowitz's theorem is only valid for a small change in conductivity within a semi-infinite homogeneous and isotropic volume conductor. Assuming the volume conductor consists of a number of discrete elements of uniform conductivity and for unit current, $\Delta Z$ is then given by

$\Delta Z=-\Delta \sigma \int_{V}[\nabla(\phi+\Delta \phi) \cdot \nabla \psi] d V=-\Delta \sigma . S$

where $\nabla \psi$ is the potential gradient before the conductivity change occurred at point $x, y, z$ due to passing of unit current between the drive electrodes $A, B$ and $\nabla(\phi+\Delta \phi)$ is the field at this point after the change occurred due to injection of unit current between the receive electrodes $C, D$ (fig. 2.1).

Both potential gradients $\nabla \varphi$ and $\nabla \psi$ are the electrical fields induced by current $I_{\varphi}$ at electrodes $A, B$ and by current $I_{\psi}$ at electrodes $C, D$ respectively. It appears that there is no analytical solution of the sensitivity $S$, which is the scalar product between two triple integrals for each coordinate $x, y$ and $z$. A finite element model can be used to get the solution ${ }^{9}$.

\section{Material and Methods}

In tetrapolar impedance measurement, it is intuitively understood that not all small sub-volumes in the material contribute equally to the measured impedance. Volumes between and close to the electrodes contribute more than volumes far away from the electrodes. Hence, a careful choice of electrode's size and placement enabled to focus measurements on the desired part of the material. Using finite element modelling, a plot of sensitivity of a given material can easily be observed, and this method provides a very valuable tool for the experimental design. The sensitivity of a small volume $d v$ within the biomaterial is a measure of how much this volume contributes to the total measured impedance ${ }^{8}$. If the resistivity varies within the material, the local resistivity must be multiplied with the sensitivity to give a measure of the volume's contribution to the total measured impedance.

For the tetrapolar impedance measurement system, the sensitivity is computed in the following ways:

1. A current, $I$ between the two drive electrodes is injected and the current density $\boldsymbol{J}_{\mathbf{1}}$ in each small volume element in the material is computed as a result of this current.

2. The same current is injected between the receive electrodes and again the resulting current density $\boldsymbol{J}_{\mathbf{2}}$ in each small volume element is computed.

3. The vector dot product of $\boldsymbol{J}_{\mathbf{1}}$ and $\boldsymbol{J}_{\mathbf{2}}$ in each volume element, divided by the current squared, is the sensitivity of the volume element and if it is multiplied with the resistivity $\rho$ in the volume, this volume's contribution to the total measured impedance $\mathrm{Z}$ is directly obtained. Hence, the sensitivity, $S$ will be as follows: 
$S=\frac{J_{1} \cdot J_{2}}{I^{2}}$

The equation (3.1) also demonstrates the reciprocal nature of the tetrapolar system - under linear conditions the drive and receive electrodes can be interchanged without changing the measured values. In this work, a cylindrical 3D model consisting of the skin-dry, muscle, fat-averageinfiltrated, bone-cortical and bone-marrow tissue layers was built using COMSOL Multiphysics 4.3 (fig. 3.1). These layers were assumed to be the replica of human upper arm anatomy. The conductivity $(\mathrm{S} / \mathrm{m})$ and relative permittivity $(\varepsilon)$ values of all these tissues were taken at $100 \mathrm{kHz}$ frequency from the literature (table 3.1) ${ }^{11}$.

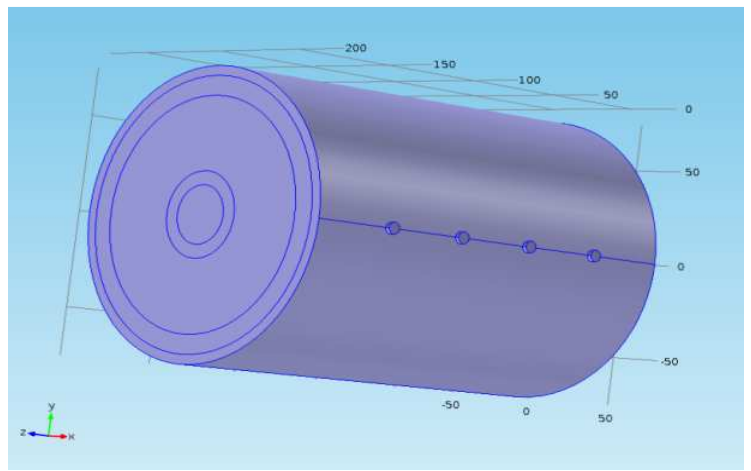

(a)

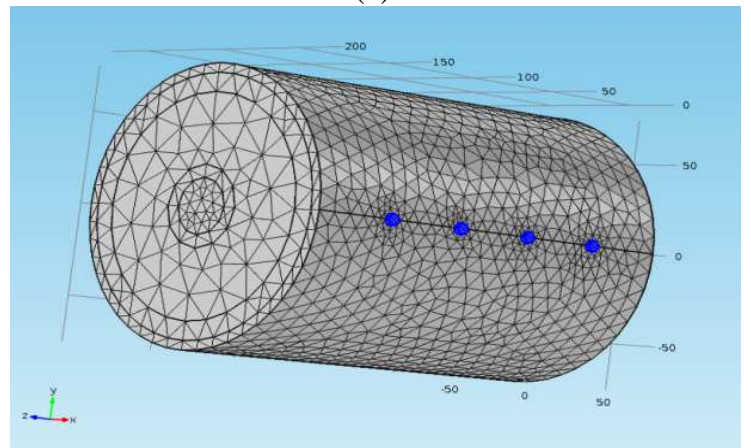

(b)

Fig. 3. 1. Showing (a) the cylindrical shaped phantom material in which conductivity is distributed non-uniformly similar to human upper hand with linearly positioned four electrodes represented as circles (b) the 3-D cylindrical model has been sectioned or meshed with triangular shaped geometrical elements.

The solid cylindrical model could easily be converted to a homogeneous media by considering all the layers having same conductivity and relative permittivity values.

In COMSOL Multiphysics, the grey scale values were replaced with tissue types or organs. This process is called segmentation. Here, the data were segmented into the most important tissue types: muscle, blood, skin-dry, fat, bonecortical, and bone-marrow. After segmentation a 3-D data set were obtained where each voxel has a name or number that represented a tissue type. When segmentation was complete, electrodes were added on the surface of cylindrical model in a linear fashion (fig. 3.1). The modelling work started with using electrodes having dimensions of $5 \mathrm{~mm}$ radius and $5 \mathrm{~mm}$ height.
Table 3.1. Electrical properties of various types of tissue in human upper hand measured at frequency $100 \mathrm{kHz}^{11}$.

\begin{tabular}{lll}
\hline Tissue & $\sigma(S / \mathrm{m})$ & $\mathcal{E}$ \\
\hline Skin Dry & 0.000045128 & 1119.2 \\
Muscle & 0.36185 & 8089.2 \\
Fat Average & 0.024414 & 92.885 \\
Infiltrated & & \\
Bone Cortical & 0.020791 & 227.64 \\
$\begin{array}{l}\text { Bone marrow } \\
\text { Infiltrated }\end{array}$ & 0.0033172 & 110.72 \\
\hline
\end{tabular}

An alternating current of magnitude 1A was injected through the drive electrode pair using the 'electric current interface' in 'AC/DC module' of COMSOL. The 3-D sensitivity distribution of tetra polar measurement was then computed using the Fred-Johan and Jan expression ${ }^{10}$ :

$$
\begin{aligned}
& \left(\left(e c \cdot J_{x}^{*} e c 2 . J_{x}+e c \cdot J_{y}^{*} e c 2 . J_{y}+e c \cdot J_{z}^{*} e c 2 . J_{z}\right)+\right. \\
& \left.\left(e c 3 . J_{x}^{*} e c 4 . J_{x}+e c 3 \cdot J_{y}^{*} e c 4 . J_{y}+e c 3 \cdot J_{z}^{*} e c 4 . J_{z}\right)\right) / \\
& \left.\left((1[A])^{\wedge} 2\right)\right)
\end{aligned}
$$

The distribution of sensitivity throughout the structure was determined in COMSOL by the finite element method (FEM) which was based on a set of partial differential equations. However, the results were an approximate solution that numerically represented the distribution of sensitivity that would be considerably difficult to obtain manually.

The graphical representation of sensitivity had been done after performing mesh by finite element method (FEM). Hence our 3-D cylindrical model had been sectioned into a number of simplistic geometric elements (e.g. triangular, tetrahedral, brick, hexahedral etc.). The collection of elements provided a discrete approximation of the object's curves in a piecewise fashion. The number of elements was finite and in turn each element had a set of known physical laws and finite parameters were applied to it. Hence, the process created a set of partial differential equations that ran simultaneously to solve the system. Here, in this work the continuous medium were subdivided into a mesh of triangular elements inside which the conductivity was assumed constant and the electric potential varied linearly. Triangular elements had been chosen for this work because of its simplicity and suitability for fitting the boundary of different conductivity regions (fig. 3.1b). In this method, the field pattern set up inside the arm and current density in each region were analysed. This numerical technique was used for both the homogenous and the inhomogeneous media. From the current density, the sensitivity was computed for both the homogeneous and inhomogeneous medium. The sensitivity distribution results for the homogeneous and inhomogeneous medium were compared with each other. In addition to this, the effect of change of electrode's dimensions on the sensitivity distributions was also checked. 


\section{Results}

The simulation results of both the homogeneous and inhomogeneous medium are presented separately.

\section{Homogenous conductivity}

The fig. 4.1 displays the sensitivity fields for our cylindrical model of homogenous conductivity with an electrode spacing of $50 \mathrm{~mm}$ and for a depth of 10 and $60 \mathrm{~mm}$ from the surface.

The sensitivity fields showed much localized areas of positive sensitivity between the receive electrode pair and negative sensitivity between the drive and receive electrode pairs at lower depth (fig. 4.1a). These regions of positive sensitivity increased with the increase of depths from the surface. However, the magnitude of sensitivity decreased substantially with the increase of depths (decreased almost $25 \%$ of the maximum for a $5 \mathrm{~mm}$ increase of depth). However, these regions of positive sensitivity started diminishing and negative sensitivity region became dominant between the receive electrode pairs for higher depths down the surface (fig. 4.1b).

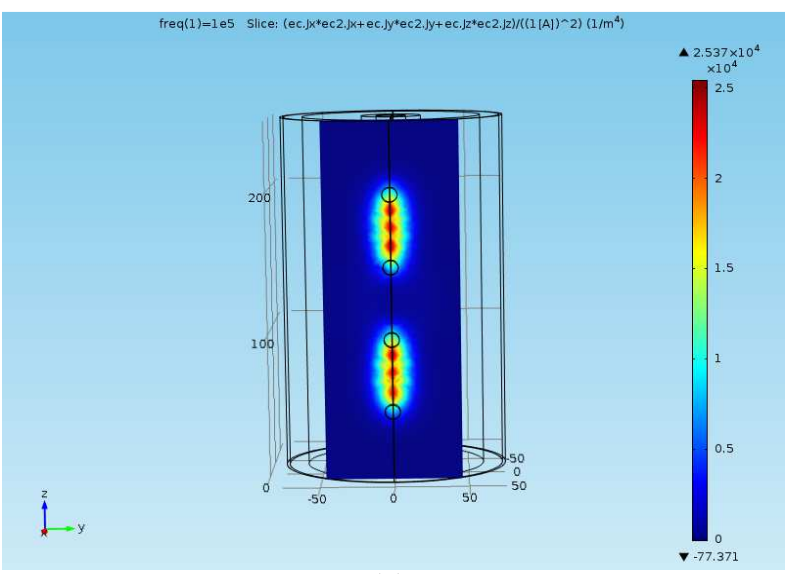

(a)

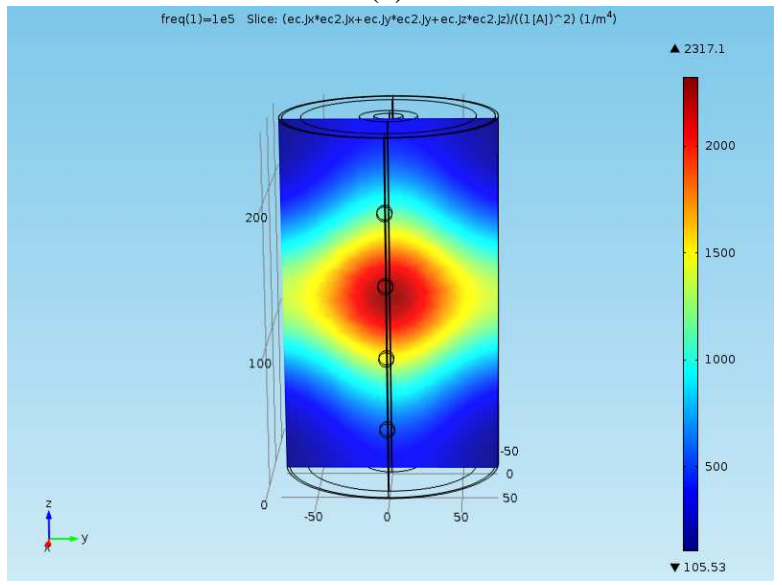

b)

Fig. 4. 1. Sensitivity distributions of the tetrapolar measurement in a uniform homogeneous conductive medium at a depth of (a) $10 \mathrm{~mm}$ and (b) $60 \mathrm{~mm}$.

The maximum sensitivity value on a plane had been found to decrease exponentially with depths (fig. 4.2) and at depth,
$90 \mathrm{~mm}$ from the surface the sensitivity had fallen to $90 \%$ of the maximum sensitivity at depth $1 \mathrm{~mm}$.

The maximum integrated sensitivity had occurred at a plane of depth approximately $1 / 3$ of drive-receive electrode spacing (fig. 4. 3).

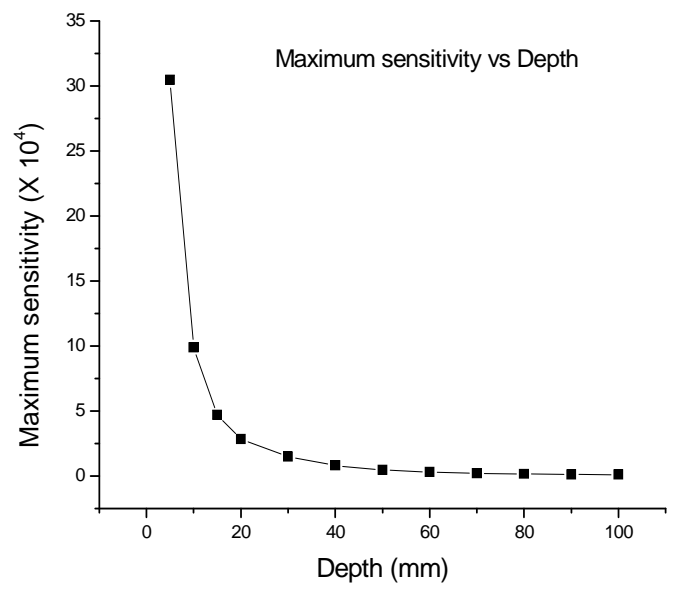

Fig. 4. 2. Shows the change of maximum sensitivity with the change of depth .

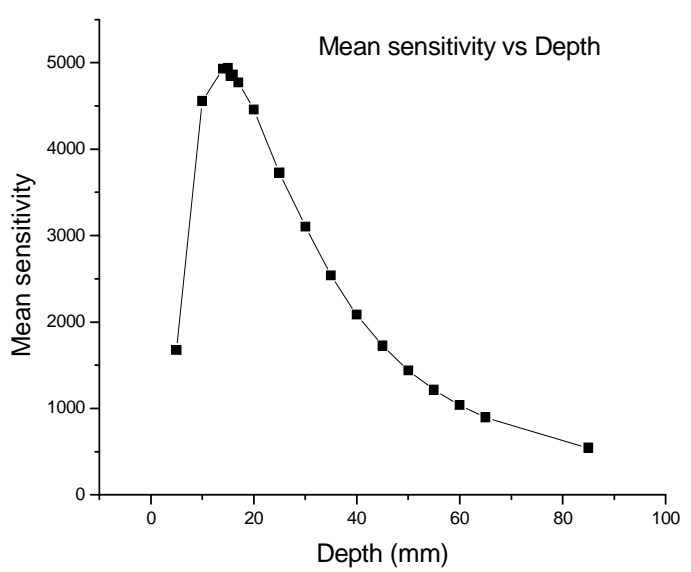

Fig. 4. 3. Shows the change of mean sensitivity with the change of depth considering drive-receive electrode spacing of $50 \mathrm{~mm}$.

The change of sensitivity with the change of electrode dimensions had also been observed. To do this, the drivereceive electrode spacing had been considered as $50 \mathrm{~mm}$ and the sensitivity was observed at a fixed depth of $15 \mathrm{~mm}$ (the depth at which maximum integrated sensitivity occurred, 1/3 of $50 \approx 15$ ) by varying the diameter of the electrode.

The fig. 4.4 shows that sensitivity changes linearly with the change of electrode dimensions. 


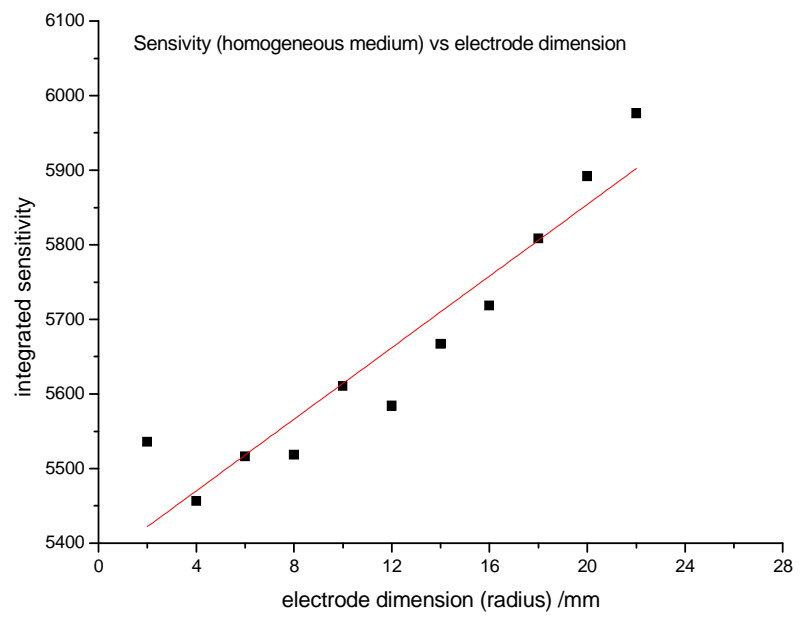

Fig. 4. 4. Shows the change of integrated sensitivity with the change of electrode dimensions for the homogeneous medium.

\section{Heterogeneous conductivity}

Here different cylinders represented different tissue layers having different conductivity values. The sensitivity distribution showed different results than those of the homogenous medium.

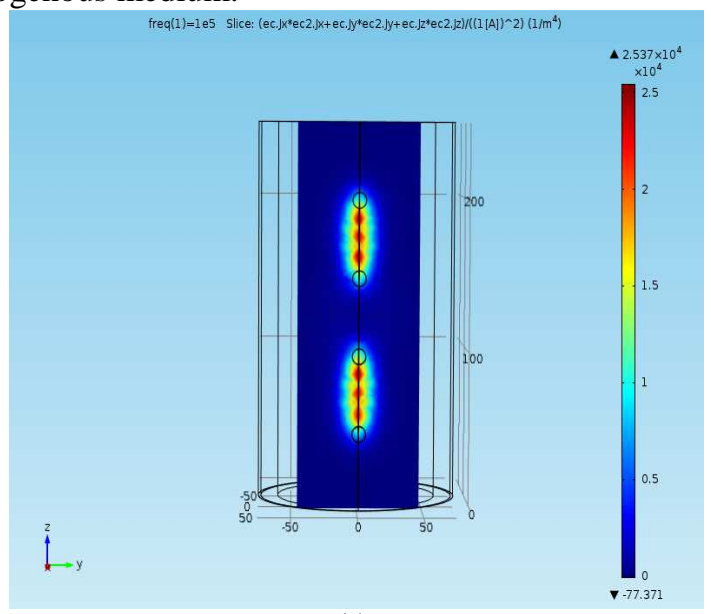

(a)

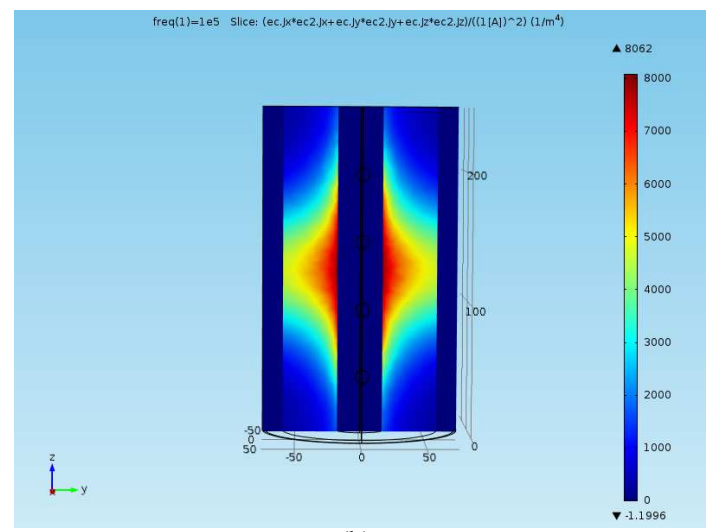

(b)

Fig. 4. 5. Shows the sensitivity distribution of the tetrapolar measurement considering a non-uniform and inhomogeneous medium at a depth of (a) 15 and (b) $65 \mathrm{~mm}$.
Fig. 4. 5 displays the sensitivity fields of inhomogeneous medium with drive-receive electrode spacing of $50 \mathrm{~mm}$ and for a depth of $10 \mathrm{~mm}$ and $60 \mathrm{~mm}$ respectively. For the inhomogeneous medium, with the increase of depth, the positive sensitivity region increased between the receive electrodes and the negative sensitivity region became dominant between the drive-receive electrodes (fig. 4. 5).

Again, fig. 4.7 shows the change of integrated sensitivity with depth with drive-receive electrode separation of $80 \mathrm{~mm}$. Here the integrated sensitivity over a plane happens to be maximum at a depth of $40 \mathrm{~mm}$. So, for the heterogeneous medium the maximum integrated or mean sensitivity occurred at half $(1 / 2)$ of the drive-receive electrode spacing. The mean sensitivity is shown here up to $100 \mathrm{~mm}$ depth. At a depth above $140 \mathrm{~mm}$ it has fallen $99 \%$ of the maximum value.

The change of integrated sensitivity with depth, with electrodes spacing of $50 \mathrm{~mm}$ is shown in fig. 4. 6. The integrated sensitivity over a plane is found maximum at a depth of $25 \mathrm{~mm}$.

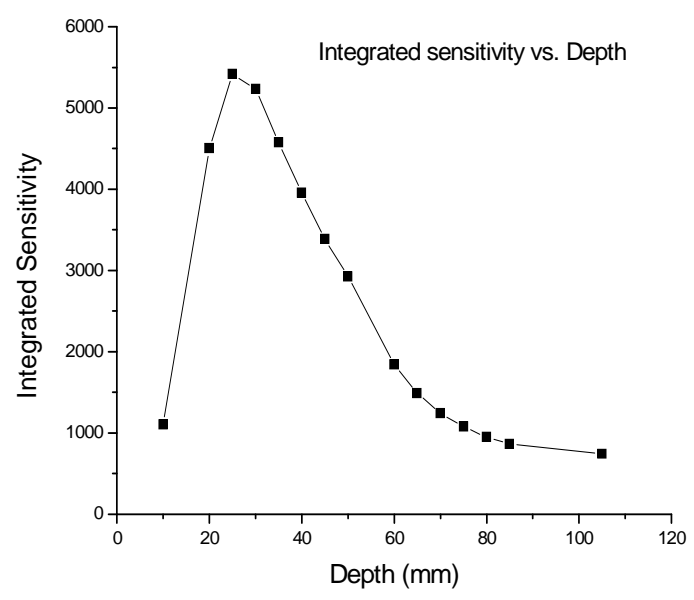

Fig. 4. 6. Maximum sensitivity shows at a depth of $25 \mathrm{~mm}$, drivereceive spacing was $50 \mathrm{~mm}$.

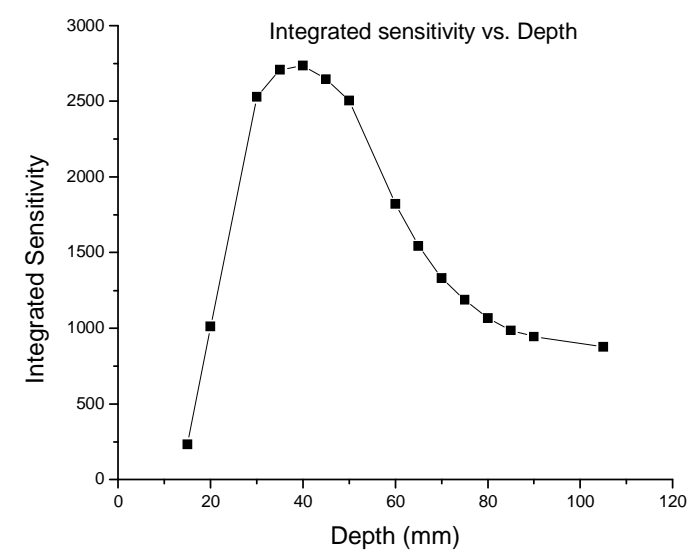

Fig. 4. 7. Shows the maximum sensitivity at $40 \mathrm{~mm}$ depth with drive-receive electrode spacing $80 \mathrm{~mm}$. 
To confirm the use of sensitivity as an indicator of measurement depth, the tetrapolar configuration was again modelled with a range of electrode separation (drivereceive) and electrode dimensions (only diameter was changed and height was kept fixed).

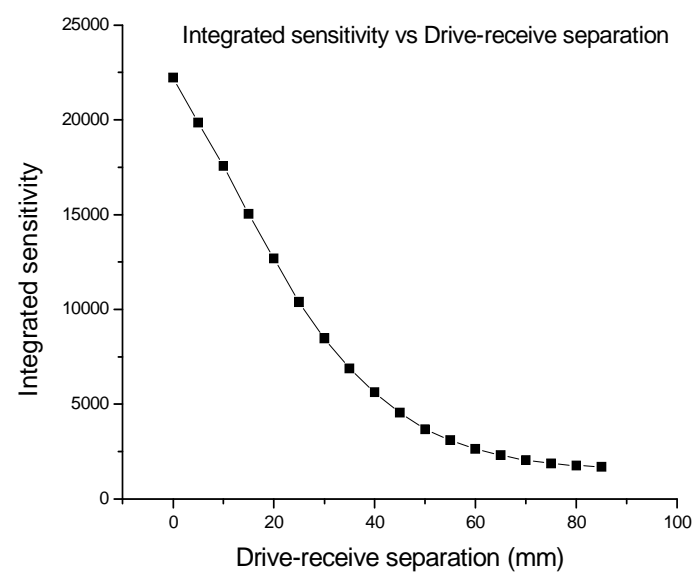

Fig. 4. 8. Integrated sensitivity vs. Drive-receive separation at a fixed $25 \mathrm{~mm}$ depth (each electrode has diameter $10 \mathrm{~mm}$ and height $5 \mathrm{~mm})$.

In fig. 4.8 , the integrated sensitivity are shown against drivereceive electode spacing at $25 \mathrm{~mm}$ depth. The integrated sensitivity decreases almost linearly for smaller drivereceive separation, then it approaches towards a constant value. In a plane the overall sensitivity decreases due to the increase of negative sensitivity region with drive-receive spacing. On the other hand, positive sensitivity region decreases with the increase of drive-receive separation.

In fig. 4.9, the integrated sensitivity is shown against the electrode dimensions at a depth of $25 \mathrm{~mm}$ and drive-receive spacing of $50 \mathrm{~mm}$. Interestingly, the change of sensitivity for both the homogenous (fig. 4.4) and inhomogeneous medium (fig. 4.9) with the change of electrodes' dimensions shows similar behaviour. For the inhomogeneous medium the sensitivity distribution also varies linearly with the electrodes' diameter.

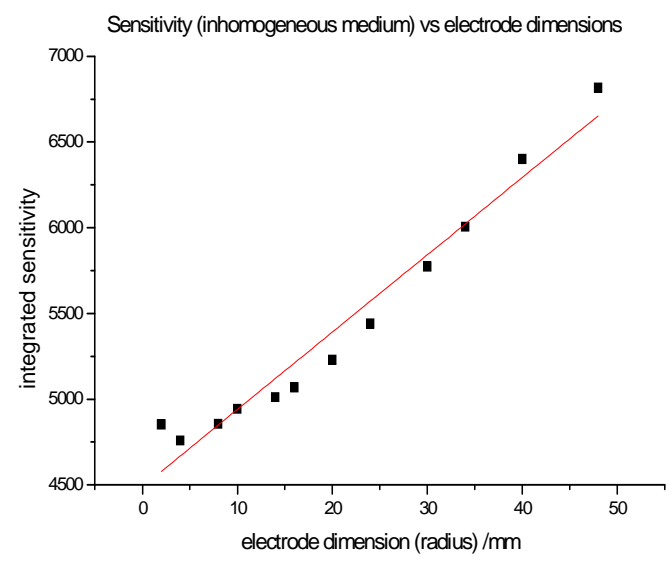

Fig. 4.9. The change of integrated sensitivity with the change of electrode dimensions for the inhomogeneous medium.

\section{Discussion}

The simulation result presented in this paper can be used to predict the positive and negative sensitivity regions which are correlated with higher and lower impedance region of an object. The previous analytical works on sensitivity analysis of homogeneous medium were done considering a simple geometry. The sensitivity distribution at a point inside a volume conductor was calculated by a programme written in MATLAB using Geselowitz lead theorem ${ }^{1,2}$. The previous works found a mean sensitivity of zero at the surface layer, a maximum average sensitivity at a plane one-third of the electrode spacing and regions of negative sensitivity down to half of the electrode spacing ${ }^{1,2}$. This work on sensitivity calculation using finite element method (FEM) confirmed those finding for homogeneous medium. However, for the inhomogeneous medium the maximum average sensitivity was found on a plane at depth half the drive-receive electrode spacing. Beyond the maximum plane, the mean sensitivity falls more slowly in the inhomogeneous medium than does in the homogenous medium. The FEM based solution of sensitivity distribution of the tetrapolar measurement of this work has also shown that the change of sensitivity with electrode dimension give similar results both for the homogenous and inhomogeneous medium. Cylindrical shaped electrodes having larger diameter provides better result in the sensitivity measurements.

\section{Conclusion}

The complex resistivity distributions of the body coupled with the complex sensitivity distribution of the tetrapolar measurement techniques have the potential to produce unrealistic estimate of transfer impedance. The sensitivity distributions obtained by finite element method (FEM) considering a complex shaped object having heterogeneous tissue structure can be considered more realistic than the previous works based on analytical method considering simple geometry done by Brown et al. ${ }^{1}$ and Islam et al. ${ }^{2}$. Moreover, if the number of element and nodes are increased by advanced finite element mesh, more accurate results could be obtained. In addition to this, multifrequency (up to $1 \mathrm{MHz}$ ) sensitivity analysis is necessary to study the complex nature of the human anatomy.

\section{References}

1. Brown, B. H., A. J. Wilson, and P. Bertemes-Filho, 2000. Bipolar and tetrapolar transfer impedance measurements from volume conductor. Electronics Letters, 36(25), 2060-2062.

2. Islam, N., K. S. Rabbani, and A. J. Wilson, 2010. The sensitivity of focused electrical impedance measurements. Physiological Measurement 31(8), S97-S109.

3. Zhang, Y., M. Qu, J. G. Webster, W. J. Tompkins, J. W. Willis, B. A. Bassett, R. David, 1986. Cardiac Output Monitoring by Impedance Cardiography during Treadmill Exercise. IEEE Transactions on, Biomedical Engineering, 33(11), 1037-1042.

4. Brown, B. H., J. A. Tidy, K. Boston, A. D. Blackett, R. H. Smallwood, and F. Sharp, 2000. Relation between tissue structure and imposed electrical current flow in cervical neoplasia. Lancet, 355(9207), 892-895. 
5. Wilson, A. J., P. Milnes, A. R. Waterworth, R. H. Smallwood, and B. H. Brown, 2001. Mk3.5: a modular, multi-frequency successor to the Mk3a EIS/EIT system. Physiological Measurement, 22(1), 49-54.

6. Brown, B. H., D. C. Barber, A. H. Morice, A. D. Leathard, 1994. Cardiac and respiratory related electrical impedance changes in the human thorax. IEEE Transactions on Biomedical Engineering, 41(8), 729-734.

7. Brown, B. H., D. C. Barber, W. Wang, L. Lu, A. D. Leathard, R. H. Smallwood, A. H. Hampshire, R. Mackayand K. Hatzigalanis, 1994. Multi-frequency imaging and modelling of respiratory related electrical impedance changes. Physiological Measurement, 15(Supple. 2A), A1-A12.
8. Geselowitz, D. B., 1971. An application of electrocardiographic lead theory to impedance plethysmography. IEEE Trans. Biomedical Engineering, BME-18(1), 38-41.

9. Murai, T. and Y. Kagawa, 1985. Electrical impedance computed tomography based on a finite element model. IEEE Trans. Biomedical Engineering, BME-32(3), 177-184.

10. Fred-Johan, P. and J. O.Hogetveit, 2011. From 3D tissue data to impedance using Simpleware ScanFE+IP and COMSOL Multiphysics - a tutorial. Journal of Electrical Bioimpedance, 2, 13-38.

11. Duck, F. A., 1990. Physical properties of tissue: a comprehensive reference book (London: Academic Press). 
